The contractile properties of standard and nerve-intact grafts of extensor digitorum longus muscles of rats were compared in vitro. Fourteen days after grafting, the time to peak tension and the half-relaxation times of nerve-intact grafts were shorter than those of standard grafts, but both were longer than control values. By 60 days, these variables attained normal values. At every sample period, the tetanic tensions of nerve-intact grafts were higher than those of standard grafts. Even at the early sampling periods, the twitch-tetanic tension ratios of nerveintact grafts were close to normal values, whereas those of standard grafts were higher than normal. Stabilized nerve-intact grafts had a larger mass and greater maximum tetanic tension development than standard grafts, but were more fatigable. Compared with control EDL muscles, stable nerve-intact grafts show no differences except for lessened fatigability, whereas standard grafts demonstrate significant functional deficits.

MUSCLE \& NERVE $\quad 8: 413-418 \quad 1985$

\title{
CONTRACTILE PROPERTIES OF STANDARD AND NERVE-INTACT MUSCLE GRAFTS IN THE RAT
}

\author{
JOHN A. FAULKNER, PhD, \\ and BRUCE M. CARLSON, MD, PhD
}

The nerve-intact model ${ }^{7}$ is a useful tool in the analysis of factors that affect the mass and functional recovery of free muscle grafts. A nerveintact graft is similar to a standard muscle graft in that both are completely removed from their beds and are completely devascularized, but the neural connections to the standard muscle graft are cut, whereas they are retained in a nerve-intact graft. In both types of grafts, all of the muscle fibers, except for a thin peripheral rim, degenerate from prolonged ischemia and subsequently regenerate. Functional neuromuscular transmission begins in standard grafts at the end of the third or early in the fourth week, whereas it is restored early in the second week in nerve-intact grafts. ${ }^{7}$ The number of skeletal muscle fibers in the standard and nerveintact grafts of the rat extensor digitorum longus

From the Departments of Physiology (Dr. Faulkner), Anatomy and Biological Sciences (Dr. Carlson), The University of Michigan, Ann Arbor, Ml.

Acknowledgments: Research was supported by USPHS Grants NS13116 and NS17017, National Institutes of Health.

Address reprint requests to Dr. Faulkner at the Department of Physiology, University of Michigan, 7775 Medical Science I| BIdg., Ann Arbor, M) 48109

Received for publication February 27,1984 : revised manuscript accepted for publication September 7, 1984.

0148-639X/0805/0413 $\$ 04.00$

c) 1985 John Wiley \& Sons, Inc
(EDL) muscle does not differ from values for control EDL muscles. In contrast to the standard grafts, which are typically restored to approximately half of normal mass and contractile force, nerve-intact grafts approach normal values for these characteristics. It has recently been shown that the greater mass and force development of nerve intact grafts cannot be attributed to the earlier restoration of the nerve supply, but is more likely due to the broader distribution of nerve fibers throughout the grafts. ${ }^{2}$

The present study was designed to document the development of contractile properties in nerveintact grafts compared with standard grafts of the EDL muscles in rats. These results on the rat will then be compared with those obtained by Faulkner et al. ${ }^{14}$ on standard and nerve-intact grafts of EDL muscles in cats.

\section{MATERIALS AND METHODS}

This experiment was carried out on 33 male Sprague-Dawley rats. At the time of muscle grafting, the rats weighed $175-200 \mathrm{~g}$. The rats were anesthetized with ether. In one leg, the EDL was completely removed from its bed and then orthotopically replaced, with both tendons sutured to their respective stumps. No attempt was made to facilitate restoration of the neural or vascular supply (standard grafts). In the other leg, the EDL 
muscle was treated identically, except that the continuity of the motor nerve was not interrupted (nerve-intact grafts). In the nerve-intact grafts, ischemia during the immediate postgrafting period results in the degeneration of intramuscular portions of the nerves. ${ }^{7}$ Grafts were evaluated at $14,20,30$, and 60 days after grafting.

Animals were anesthetized and the EDL muscles or grafts were isolated and removed. The muscles or grafts were attached to a force transducer and immersed in a mammalian Krebs buffered bicarbonate solution. ${ }^{9}$ Isometric contractile properties were measured in vitro at $37^{\circ} \mathrm{C}$. The muscles and grafts were stimulated directly with two platinum field electrodes. Stimulation was by square-wave pulses of $0.2 \mathrm{msec}$ duration and supramaximum voltage. The variables measured were: time to peak twitch tension (TPT) and halfrelaxation time (RT 1/2), maximum twitch tension $\left(\mathrm{P}_{\mathrm{t}}\right)$, maximum tetanic tension $\left(\mathrm{P}_{\mathrm{o}}\right)$, and the optimal muscle length $\left(\mathrm{L}_{\mathrm{o}}\right)$ for tension development. ${ }^{15}$

Muscle lengths were measured at $\mathrm{L}_{o}$, and fiber lengths were estimated based on a fiber length/ muscle length ratio of $0.4 .^{11}$ The cross-sectional area was calculated from mass, fiber length, and a muscle density of $1.06 .^{15}$ The fatigability of grafts was measured during a sustained contraction at $100 \mathrm{~Hz}$ lasting $10 \mathrm{sec}$. The tension at $10 \mathrm{sec}$, expressed as a percentage of the maximum tension, was used as an index of fatigue.

Significant differences between standard and nerve-intact grafts and between grafts and control EDL muscles in age-matched animals at 14 and 60 days after grafting were determined by $t$ tests. The level of significance was set at $1 \%$.

\section{RESULTS}

The rats were $47-50$ days of age at the time of grafting. During the 60-day postgrafting period, the body mass of the rats increased from 267 to $453 \mathrm{~g}$ and the mass of the control EDL muscle from 137 to $221 \mathrm{mg}$. The RT $1 / 2$ was stabilized at $9-10 \mathrm{msec}$ by 50 days, but the TPT increased slightly from $10 \mathrm{msec}$ to $12 \mathrm{msec}$ between 50 and 160 days of postnatal development. The $\mathrm{P}_{\mathrm{t}} / \mathrm{P}_{\mathrm{o}}$ decreased significantly. These results are in agreement with previous observations of developing EDL muscles in rats. ${ }^{5,9}$

Morphological Variables. During the 60 -day postgrafting period, the mean value for body mass of the animals increased by $64 \%$ (Table 1). The mass of the nerve-intact grafts was greater than that of the standard grafts at each sample period. The dif- ference ranged from $30 \%$ to $60 \%$. Between 14 and 60 days after grafting, the mass of the grafts more than doubled, whereas the control muscle increased its mass by $70 \%$. Sixty days after grafting, the mass of the standard grafts was $59 \%$ of the control value and that of the nerve-intact grafts was $87 \%$.

The muscle length of age-matched control EDL muscles increased by $30 \%$ between days 14 and 60 after grafting (Table 1). On day 14, the standard and nerve-intact grafts had muscle lengths $80 \%$ and $87 \%$, respectively, of the control value. On day 60 , the muscle lengths of both types of grafts were $80 \%$ of the control EDL muscle. No significant differences were observed between the muscle lengths of the two types of grafts.

As with mass, the total cross-sectional area of the nerve-intact grafts was significantly greater than that of the standard grafts at each time point. Sixty days after grafting, the cross-sectional area of the standard grafts was $48 \%$ less than the control value. The $4 \%$ difference between the area of nerve-intact grafts and control EDL muscles was not statistically significant.

Contraction and Relaxation Times. During the early sample periods, the values for TPT of both standard and nerve-intact grafts were significantly prolonged in comparison with the values for TPT' of control muscles (Table 2), but at 14 days, the TPT of nerve-intact grafts was already significantly shorter than that of standard grafts. Following the typical pattern of maturation for regenerating rat muscle, the TPTs rapidly became shorter during the first 30 days and returned to normal values by 60 days. The development of RT $1 / 2$ followed a pattern similar to that of the TPT.

Twitch and Tetanic Tension (Tables 1 and 2). At 14 days, the $P_{t}$ of standard grafts was greater than that of nerve-intact grafts, but with time, the $P_{t}$ of the nerve-intact grafts increased to higher levels than did those of standard grafts. At 60 days, the value for $\mathrm{P}_{\mathrm{t}}$ of standard grafts was $50 \%$ of the control value and that of the nerve-intact grafts was $73 \%$. During the early postgrafting period, the $\mathrm{P}_{0}$ of the nerve-intact grafts rose more rapidly than the $P_{o}$ of standard grafts (Fig. 1). At 60 days, the $P_{o}$ of standard and nerve-intact grafts was $57 \%$ and $91 \%$ of the control value, respectively. During the first 30 days after grafting, the $P_{t}$ of the standard grafts showed a greater increase relative to the control value than did $P_{o}$, whereas the values for the $P_{t}$ and $P_{o}$ of the nerve-intact grafts increased in 
Table 1. The morphological characteristics of extensor digitorum longus (EDL) muscles, standard (STD) grafts, and nerve-intact ( $\mathrm{N}-1)$ grafts

\begin{tabular}{|c|c|c|c|c|}
\hline & $\begin{array}{c}\text { Animal mass } \\
(\mathrm{g})\end{array}$ & $\begin{array}{l}\text { Muscle mass } \\
\text { (mg) }\end{array}$ & $\begin{array}{l}\text { Muscle length } \\
\quad(\mathrm{mm})\end{array}$ & $\begin{array}{c}\text { Muscle cross- } \\
\text { sectional area } \\
\qquad\left(\mathrm{mm}^{2}\right)\end{array}$ \\
\hline \multicolumn{5}{|l|}{ 14-Day control } \\
\hline $\begin{array}{l}\mathrm{EDL}(N=5) \\
14 \text {-Day grafts }\end{array}$ & $267 \pm 6$ & $137.2 \pm 6.6$ & $29.4 \pm 0.9$ & $11.0 \pm 0.6$ \\
\hline$(N=8)$ STD & $276 \pm 8$ & $62.3 \pm 6.4^{*}$ & $23.5 \pm 0.7^{*}$ & $6.3 \pm 0.7^{*}$ \\
\hline$(N=8) N-1$ & $276 \pm 8$ & $80.6 \pm 4.0^{\star} \dagger$ & $25.5 \pm 1.7$ & $7.7 \pm 0.6^{\star}$ \\
\hline \multicolumn{5}{|l|}{ 20-Day grafts } \\
\hline$(N=8)$ STD & $295 \pm 26$ & $80.6 \pm 4.0$ & $27.8 \pm 0.7$ & $6.8 \pm 0.4$ \\
\hline$(N=8) \mathrm{N}-\mathrm{I}$ & $295 \pm 26$ & $130.5 \pm 6.0 \dagger$ & $31.5 \pm 0.5 \dagger$ & $10.2 \pm 0.4 \dagger$ \\
\hline \multicolumn{5}{|l|}{ 30-Day gratts } \\
\hline$(N=8)$ STD & $291 \pm 8$ & $72.5 \pm 11.0$ & $23.5 \pm 1.0$ & $6.6 \pm 0.9$ \\
\hline$(N=8) N-1$ & $291 \pm 8$ & $113.2 \pm 10.0 \dagger$ & $25.0 \pm 0.7$ & $10.6 \pm 1.0 \dagger$ \\
\hline \multicolumn{5}{|l|}{ 60-Day grafts } \\
\hline$(N=8)$ STD & $422 \pm 14$ & $131.1 \pm 17.9^{\star}$ & $30.8 \pm 1.0$ & $10.4 \pm 1.4^{*}$ \\
\hline$(N=8) N-1$ & $422 \pm 14$ & $192.4 \pm 9.9+$ & $30.8 \pm 0.7$ & $14.8 \pm 0.8 \dagger$ \\
\hline \multicolumn{5}{|l|}{ 60-Day control } \\
\hline $\operatorname{EDL}(N=4)$ & $453 \pm 18$ & $221.0 \pm 17.5$ & $38.5 \pm 3.2$ & $15.4 \pm 1.3$ \\
\hline
\end{tabular}

Significant differences $(P<0.05)$ between grafts and control EDL muscles at 14 days and 60 days are indicated by an asterisk $\left(^{*}\right)$ and between nerve-intact and standard grafts at the same time period by a dagger $(t)$ Comparisons were not made between grafts and control muscles 20 and 30 days after grafting. Data are for means \pm 1 SEM

concert. The result of these differences was significantly higher $\mathrm{P}_{\mathrm{t}} / \mathrm{P}_{\mathrm{o}}$ ratios for the standard grafts than for the nerve-intact grafts during the early postgrafting period. By 60 days, the $\mathrm{P}_{\mathbf{r}} / \mathrm{P}_{\mathrm{o}}$ ratios of both groups had reached control values.

The specific tensions (tension in Newtons normalized per square centimeter of cross-sectional area) of the nerve-intact grafts were greater than those of the standard grafts at each sample period.
The specific tensions for both types of grafts increased significantly with time after grafting. At 60 days, the specific tension of the nerve-intact grafts was not significantly different from the control value.

Frequency-Force Relationship. The frequency-force curve of the standard grafts was shifted upwards and to the left to a considerably greater degree

\begin{tabular}{|c|c|c|c|c|c|c|}
\hline & $\begin{array}{c}\text { TPT } \\
\text { (msec) }\end{array}$ & $\begin{array}{l}\mathrm{RT} 1 / 2 \\
\text { (msec) }\end{array}$ & $\begin{array}{c}P_{t} \\
(\mathrm{mN})\end{array}$ & $\begin{array}{c}P_{0} \\
(\mathrm{mN})\end{array}$ & $\begin{array}{c}P_{0} \\
\left(N / \mathrm{Cm}^{2}\right)\end{array}$ & $P_{t} / P_{0}$ \\
\hline \multicolumn{7}{|l|}{ Control EDL } \\
\hline$(N=5)$ & $10.3 \pm 0.5$ & $11.1 \pm 1.0$ & $424 \pm 26$ & $1958 \pm 122$ & $18.0 \pm 1.0$ & $0.22 \pm 0.01$ \\
\hline \multicolumn{7}{|l|}{ 14-Day grafts } \\
\hline$(N=8)$ STD & $22.3 \pm 0.4^{\star}$ & $17.8 \pm 0.8^{\star}$ & $142 \pm 10^{*}$ & $276 \pm 23^{\star}$ & $4.8 \pm 0.6^{\star}$ & $0.53=0.03^{\star}$ \\
\hline$(N=8) N-I$ & $15.9 \pm 0.7^{\star} \dagger$ & $16.3 \pm 1.0^{*}$ & $120 \pm 10^{*}$ & $541 \pm 48^{\star} \dagger$ & $7.2 \pm 0.9^{*} \dagger$ & $0.22 \pm 0.01 \dagger$ \\
\hline \multicolumn{7}{|l|}{ 20-Day grafts } \\
\hline$(N=8)$ STD & $22.1 \pm 1.0$ & $20.1 \pm 0.7$ & $133 \pm 27$ & $331 \pm 88$ & $5.7 \pm 0.9$ & $0.35 \pm 0.04$ \\
\hline$(N=8) N-1$ & $14.9 \pm 0.5 \dagger$ & $14.7 \pm 1.0 \dagger$ & $180 \pm 32$ & $1010 \pm 117 \dagger$ & $10.0 \pm 1.0+$ & $0.18 \pm 0.02 \dagger$ \\
\hline \multicolumn{7}{|l|}{ 30-Day grafts } \\
\hline$(N=8) S T D$ & $13.7 \pm 1.3$ & $16.6 \pm 2.0$ & $118 \pm 21$ & $386 \pm 100$ & $6.2 \pm 0.8$ & $0.39 \pm 0.05$ \\
\hline$(N=8) N-1$ & $9.5 \pm 0.2 \dagger$ & $8.1 \pm 1.0 \dagger$ & $221 \pm 22 \dagger$ & $1285 \pm 145 \dagger$ & $11.4 \pm 0.8 \dagger$ & $0.18 \pm 0.01 \dagger$ \\
\hline \multicolumn{7}{|l|}{ 60-Day grafts } \\
\hline$(N=8)$ STD & $12.2 \pm 0.4$ & $11.2 \pm 0.5$ & $202 \pm 51^{*}$ & $1355 \pm 261^{*}$ & $10.4 \pm 1.7^{\star}$ & $0.15 \pm 0.02$ \\
\hline$(N=8) N-1$ & $12.1 \pm 0.2$ & $10.1 \pm 0.3$ & $291 \pm 17^{\star}$ & $2151 \pm 89 \dagger$ & $13.6 \pm 0.9$ & $0.14 \pm 0.01$ \\
\hline \multicolumn{7}{|l|}{ Control EDL } \\
\hline$(N=4)$ & $12.0 \pm 0.3$ & $9.9 \pm 0.5$ & $400 \pm 31$ & $2367 \pm 128$ & $15.5 \pm 0.9$ & $0.17 \pm 0.01$ \\
\hline
\end{tabular}

Significant differences $(P<0.05)$ between grafts and control EDL muscles at 14 days and 60 days are indicated by an asterisk (") and between nerve-intact and standard grafts at the same time period by a dagger ( $t$ ) Comparisons were not made between grafts and control muscles 20 and 30 days after grafting. Data are for means \pm 1 SEM. 


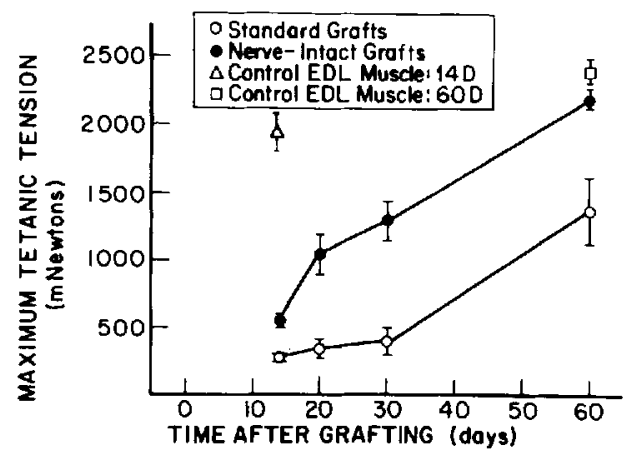

FIGURE 1. The maximum isometric tetanic tension of control EDL muscles, standard grafts, and nerve-intact grafts at time periods from 14 to 60 days after grafting. Means \pm 1 SEM are indicated.

than the frequency-force curve of the nerve-intact grafts (Fig. 2). The frequency-force curve of the nerve-intact grafts returned to the control relationship by 30 days after grafting. The frequencyforce curve of the standard grafts did not achieve the control relationship until 60 days after grafting.

Fatigability. The fatigability of nerve-intact grafts was quite different from that of the standard grafts at all sampling periods after grafting (Fig. 3). The nerve-intact grafts maintained a fairly constant fatigue index of from $41 \%$ to $51 \%$ throughout the 60 -day postgrafting period. At 14 days postgrafting, the standard grafts had a fatigue index of $15 \%$, which increased to $62 \%$ at 60 days (Fig. 3). At 14 days postgrafting, the fatigue index of the stan-

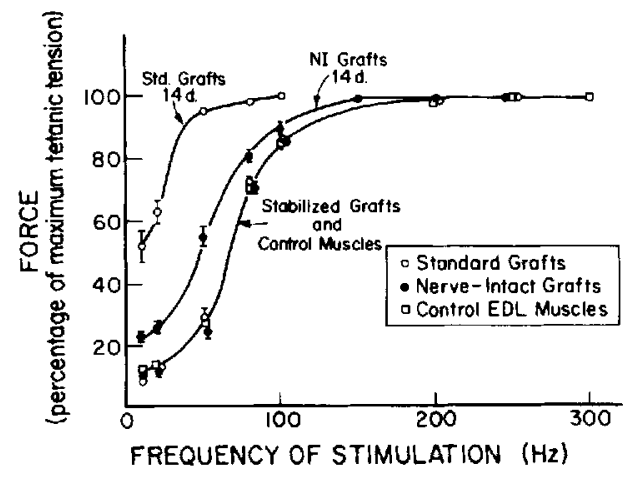

FIGURE 2. The frequency-force curves for control EDL muscles and for standard and nerve-intact grafts at selected time periods after grafting. The standard grafts $(\rho)$ and nerveintact grafts $(\bullet)$ are shown at 14 days after grafting (upper left plots) and after return to control values by 30 days for the nerve-intact grafts and by 60 days for the standard grafts (lower right plots). The means \pm 1 SEM are indicated. Some error bars are within symbols.

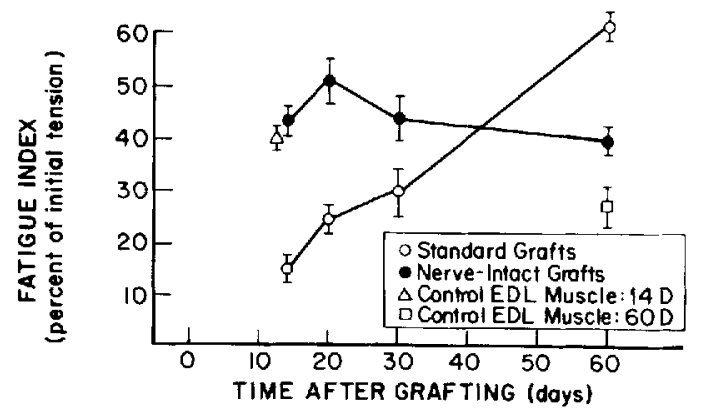

FIGURE 3. The fatigue indices of control EDL muscles, standard grafts, and nerve-intact grafts at time periods from 14 to 60 days after grafting. Means \pm 1 SEM are indicated.

dard grafts was significantly less than the control value, whereas that of nerve-intact grafts was not different from that of the control EDL muscles. At 60 days, both standard and nerve-intact grafts were less fatigable than the control muscles. The $\mathrm{P}_{0}$ provides an effective estimate of the viable crosssectional area of muscle fibers in muscles, and particularly in grafts that have large amounts of connective tissue. ${ }^{14,15}$ The fatigue indices of small and large EDL muscles and grafts were plotted against maximum tetanic tension to evaluate the role viable cross-sectional area of muscle fibers plays in the development of fatigue (Fig. 4). No clear overall relationship was apparent. The poorly developed 14-, 20-, and 30-day standard grafts and large mature control muscles had short fatigue indices, but with low and high values for $P_{0}$, respectively. The 60-day standard grafts, all nerve-intact grafts, and small immature control muscles had prolonged fatigue indices in spite of a wide range of $\mathrm{P}_{\mathrm{o}} \mathrm{s}$.

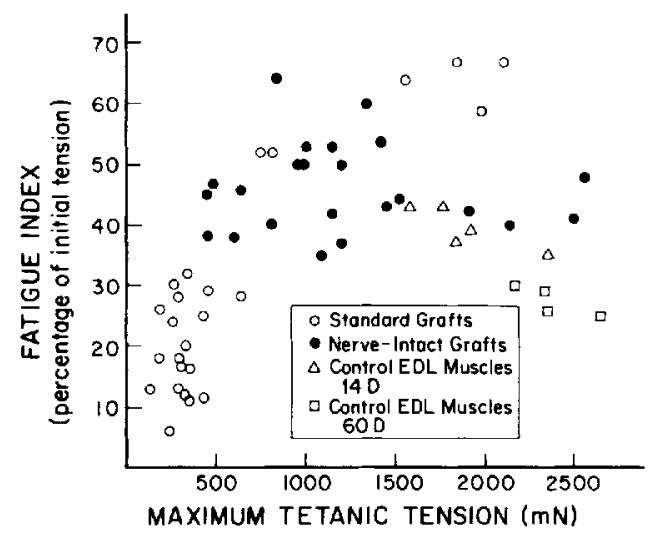

FIGURE 4. The fatigue indices of individual control EDL muscles, standard grafts, and nerve-intact grafts plotted against maximum isometric tetanic tension. 


\section{DISCUSSION}

A comparison of the development of standard with nerve-intact muscle grafts leads to three major generalizations. The first is that the contraction and relaxation times and frequency-force relationships of both types of grafts ultimately return to control values. The second generalization is that, in keeping with their greater mass and crosssectional area, nerve-intact grafts develop greater maximum tension than do standard grafts. Lastly, at 60 days after grafting, both types of grafts demonstrate a greater resistance to fatigue than control EDL muscles.

Previous work ${ }^{3,5}$ has shown that immature regenerating muscles in rats have a prolonged TPT, but that by about 40 days, control values are reached. During the first 3-4 weeks after grafting, the TPT of the regenerating muscle fibers shortens considerably in the presence or absence of a functional innervation. This myogenic maturation of muscle function occurs even in permanently denervated regenerating muscle. ${ }^{6}$ This pattern of maturation is reflected in the present data for standard grafts.

Nerve-intact grafts are innervated by $7-14$ days, ${ }^{7}$ and the contractile data for the nerve-intact grafts reflect the influence of a functional innervation superimposed upon the intrinsic maturation of the regenerating muscle fibers. This is shown in the shorter time for contraction throughout the periods studied, but an even stronger indication is seen in the $\mathrm{P}_{\mathrm{t}} / \mathrm{P}_{\mathrm{o}}$. Immature muscle, whether in normal ontogenesis or during regeneration, is characterized by a $\mathrm{P}_{t} / \mathrm{P}_{\mathrm{o}}$ close to $0.5 .{ }^{9}$ As the muscle matures, the ratio approaches $0.15-0.25$, depending on the muscle and the species. ${ }^{9,10}$ The pronounced difference in $P_{t} / P_{o}$ between standard and nerve-intact grafts at $14-30$ days postgrafting is probably the best indicator of the maturationenhancing effect of the nerve supply on regenerating muscle. By the time grafts in rats or cats ${ }^{14}$ are stable, no differences are observed in contraction or relaxation times or in frequency-force relationships. We conclude that the beneficial effect of the nerve-intact procedure for grafting lies elsewhere than in the contractile machinery of mature regenerated muscle fibers.

A major difference between small 100-mg grafts in rats and large $3-\mathrm{g}$ grafts in cats is in the functional mass and number of muscle fibers that regenerate in grafts of rats and cats. In the rat, the mass and numbers of muscle fibers in nerve-intact grafts are restored to essentially normal values, and the $\mathrm{P}_{\mathrm{o}}$ is close to $90 \%$ of control value. ${ }^{7}$ Con- versely, in the cat, both mass and number of muscle fibers are below normal values, and the $P_{o}$ attains only $67 \%$ of the normal value. ${ }^{14}$

The specific tension of a variety of control muscles from different species range from 15 to $30 \mathrm{~N} /$ $\mathrm{cm}^{2} .{ }^{10}$ Our values for control EDL muscles are in the lower range of these values. Muscles that have contracted vigorously over a period of hours have a mass approximately $20 \%$ greater than rested muscles. ${ }^{15,16}$ Furthermore, EDL muscles larger than $50 \mathrm{mg}$ do not sustain $\mathrm{P}_{\mathrm{o}}$ over time when tested at $37^{\circ} \mathrm{C}$ in vitro. ${ }^{17}$ We have reported values for specific tension of $28 \mathrm{~N} / \mathrm{cm}^{2}$ for both EDL grafts and EDL muscles in cats. ${ }^{14,15}$ The low value for specific tension of control muscles results from a decline in maximum tension with time at $37^{\circ} \mathrm{C}$ in vitro and the increased fluid volume.

Lower values for specific tensions of grafts when compared to control EDL muscles have been reported previously in rats ${ }^{11}$ and cats. ${ }^{14,15}$ The low specific tensions of grafts have been accounted for previously by an increased content of noncontractile tissue in grafts. ${ }^{15}$ The higher specific tensions for the nerve-intact grafts indicate relatively more contractile tissue and less connective tissue in nerve-intact than in standard grafts.

The second generalization concerns fatigability. The fatigability of the small EDL grafts in rats is complex. ${ }^{12}$ The standard grafts in rats are initially more fatigable than control EDL muscles. The mechanism for the rapid fatigue of the small immature standard grafts is not a function of mass. A significant factor may be the relative tension development at $100 \mathrm{~Hz}$. The 14-day standard grafts are developing maximum tetanic tension, whereas control muscles and other grafts range from $75 \%$ to $90 \%$ of maximum (Fig. 2). By 60 days, both types of grafts have longer fatigue indices than control EDL muscles. Stabilized standard and nerve-intact EDL grafts of rats have demonstrated a greater resistance to fatigue than control EDL muscles with various in situ tests of fatigability. ${ }^{8,11}$ Compared to control EDL muscles, the grafts have the same number, but smaller, fibers, ${ }^{1,7}$ higher capillary density, ${ }^{8}$ and a higher concentration of cytochrome-c. ${ }^{8}$ These characteristics of the small EDL grafts in rats might explain the high resistance to fatigue measured in situ, but they do not account for an increased resistance to fatigue in vitro.

The increased resistance to fatigue of mature grafts in rats contrasts with the data on large, standard and nerve-intact grafts in cats that are more fatigable than control EDL muscles at all time pe- 
riods up to a year after grafting. ${ }^{14,15,18}$ The difference in the fatigability of small grafts in rats and large grafts in cats may result from the differences in the number, cross-sectional area, and type of fibers that regenerate. During the 10 -sec in vitro fatigue protocol, the resistance to fatigue should be a function of the total available energy, the rate of energy utilization, the build-up of metabolic endproducts, or some combination of these factors. ${ }^{13}$ The exact mechanism of fatigue remains unknown. ${ }^{12}$ The major differences in the fatigability of standard grafts at different time periods after grafting and between the fatigability of small 100 $\mathrm{mg}$ and large 3-g grafts offers a unique opportunity to study the factors responsible for fatigue.

Lastly, the main difference between the degree of recovery achieved by nerve-intact compared to the standard grafts is the larger total functional mass of the former. Evaluation of the two primary factors limiting the functional mass attained by muscle grafts indicates that interruption of the vascular supply is not critical, but interruption of the nerve supply to grafts of 100-150-mg EDL muscles in the rat results in a significant functional deficit. Although the return of the blood supply does not appear to play a significant role in limiting the mass of muscle that regenerates in these small grafts, more complete innervation may indirectly influence revascularization. In contrast, maintaining the nerve intact was not sufficient for the recovery of full functional mass in the larger EDL grafts in the cat. Apparently, some aspect of the return of the vasculature, either the timing or extent of revascularization, plays a more important role in the larger grafts. By standardizing the degree of reinnervation, the nerve-intact procedure allows one to investigate other factors, such as revascularization or the mechanical environment, as variables that could influence the restoration of structure and function of a muscle graft.

\section{REFERENCES}

1. Cartson BM: A quantitative study of muscle fiber survival and regenerating in normal. predenervated and Marcainetreated free muscle grafts in the rat. Exp Neurol 52:421$432,1976$.

2. Carlson BM, Foster AH, Bader DM, Hník P, Vejsada R: Restoration of full mass in nerve-intact muscle grafts after delayed reinnervation. Experientia 39:171-172, 1983.

3. Carlson BM, Gutmann E: Development of contractile properties of minced muscle regenerates in rats. Exp Neurol $36: 239-249,1972$

4. Carlson BM, Gutman E: Reyeneration in free gratis of normal and denervated muscles in the rat: morphology and histochemistry. Anut Rec 183:47-62, 1975.

5. Carlson BM, Gutmann E: Regeneration in grafts of normal and denervated rat muscles: contractile properties. Pfluegers Arch 353:215-225, 1975.

6. Carlson BM, Gutmann E: Contractile and histochemical properties of sliced muscle grafts regenerating in normal and denervated rat limbs. Exp Neurol 50:319-329, 1976.

7. Carlson BM, Hník P, Tuček S, Vejsada R, Bader D, Faulkner $J A$ : Comparison between grafts with intact nerves and standard free grafts of the rat extensor digitorum longus muscle. Physiol Bohemoslov 30:505-513, 1981 .

8. Ciske PE, Faulkner JA: Chronic stimulation of extensor digitorum longus (EDL) grafts in rats. Med Sci Sports Med $15: 131,1983$.

9. Close RI: Dynamic properties of fast and slow skeletal muscles of the rat during development. $J$ Physiol (London)

$173: 74-95,1964$

10. Close RI: Dynamic properties of muscle. Physiol Rev 52:129-197, 1972.

11. Côté C, Faulkner JA: Motor unit function in skeletal muscle autografts of rats. Exp Neurol 84:292-305, 1984.

12. Edwards RHT: Human muscle function and fatigue, in Porter R, Whelan J (eds): Human Muscle Fatigue: Physiological Mechanisms, Ciba Foundation Symposium 82. London, Pitman Medical Lid., 1981, pp 1-18.

13. Edwards RHT, Hill DK, Jones DA: Heat production and chemical changes during isometric contractions of the human quadriceps muscle. I Physiol (Lond) 251:303-315, 1975.

14. Faulkner JA, Markley JM Jr, MCCully KK, Watters CR, White TP: Characteristics of cat skeletal muscles grafted with intact nerves or with anastomosed nerves. Exp Neurol 80:682-696, 1983.

15. Faulkner JA, Niemeyer JH, Maxwell LC, white TP: Contractile properties of transplanted extensor digitorum longus muscles of cats. Am J Physiol 238:C120-C126, 1980.

16. Murphy RA, Beardsley AC: Mechanical properties of the cat soleus muscle in situ. Am J Physiol 227:1008-1013, 1974.

17. Segal S, Faulkner JA: Status endurance of rat skeletal muscles at different temperatures in vitro. Med Sci Sports Exercise 14:106, 1982.

18. White TP, Maxwell LC, Sosin DM, Faulkner JA: Capillarity and blood flow of transplanted skeletal muscles of cats. $A m$ $J$ Physiol 241:H630-H636, 1981. 